\title{
NORDISCH - GERMANISCH - DEUTSCH? ZUR MythenRezeption IM Heavy Metal
}

\author{
Florian Heesch
}

Sucht man nach dem »typisch Deutschen « in der Musik und begrenzt sich dabei versuchsweise einmal nicht auf die populäre Musik im engeren Sinn, wäre Richard Wagners Operntetralogie Der Ring des Nibelungen nicht ein hervorragendes Beispiel? Stehen nicht gerade die wagnerschen Götter (Wotan) und Helden (Siegfried), die Walküren und Nornen, die von Riesen erbaute Götterburg Walhall oder das Untergangszenario der Götterdämmerung für mythologische Elemente einer deutschen Kultur? Das lässt sich dann bejahen, wenn man etablierten Klischees von der deutschen Identität jener Mythen und Heldensagen folgt - Klischees, die sich durchaus nicht nur in der sogenannten Hochkultur, sondern auch in Alltagskultur und populärer Kultur wiederfinden. Wie verhält es sich folglich mit dem Verhältnis zu deutscher Identität, wenn heute, circa 150 Jahre nach Wagner, zahlreiche Heavy Metal-Bands Elemente aus der nordischen Mythologie und Heldensage in ihren Songs verarbeiten? Inwiefern knüpft diese Musik, ein Teil der populären Musik des 20. und 21. Jahrhunderts, an stereotype Deutungen jener Mythologie als eine >germanische< bzw. >deutsche< an? Inwiefern befördert solcher Mythen-Metal möglicherweise entsprechende Mythendeutungen oder gar nationalistische Ideen? Eine Untersuchung der Mythenrezeption im Heavy Metal kann, wie ich meine, zur Reflexion darüber beitragen, was in der populären Musik heute als das Deutsche identifiziert wird. Denn man stößt dabei nicht nur auf eine durchaus heterogene Vielfalt rockmusikalischer Mythenbezüge, auf eine häufig interessante Bandbreite an stilistischen Umsetzungen, Verflechtungen mit anderen Themen und teils komplexen Kombinationen aus Musik, Texten und Bildern, sondern es geht zentral auch um die Frage, wie deutsche Identität konstruiert wird, stehen doch hinter den zitierten Klischees von vermeintlich deutschen Götter- und Heldenfiguren bestimmte kulturelle Konstrukte.

Diese Konstrukte lassen sich in Form zweier Gleichungen abbilden, nämlich erstens in der Gleichung »germanisch = deutsch« und zweitens in der 
Auffassung von der nordischen Mythologie als germanischer. Daran, dass diese Gleichungen in der deutschen Kultur außerhalb wissenschaftlicher Spezialdiskurse bis heute selten infrage gestellt werden, hat unter anderem der anfangs erwähnte Ring des Nibelungen von Wagner einigen Anteil. Im ersten Teil des vorliegenden Beitrags werde ich kurz skizzieren, wie sich die genannten Konstrukte kulturhistorisch entwickelten, was im vorgegebenen Rahmen notwendigerweise auf einen kurzen Überblick begrenzt werden muss. Auf dieser Grundlage werde ich dann drei Beispiele deutscher MetalBands diskutieren, die exemplarisch für verschiedene Zugänge zur nordischen Mythologie und für verschiedene Bezugnahmen auf Konstrukte von germanischer und deutscher Identität stehen.

Dass das Interesse an nordischen Mythen und Heldensagen heute vor allem im Heavy Metal weit verbreitet ist (vgl. Bénard 2004; von Helden 2012), lässt sich schon durch einen Blick auf Bandnamen oder Lyrics leicht feststellen. Bei einer Recherche nach nordischen Mythologemen (z.B. Asgard, Einheriar, Fenrir sowie variante Schreibungen) in der umfangreichen Datenbank Encyclopaedia Metallum (http://www.metal-archives.com) fanden wir 2011 knapp 600 verschiedene Bands weltweit, deren Namen sich aus der nordischen Mythologie ableiten. ${ }^{1}$ Nicht immer bedeutet ein solcher Name, dass die dahinterstehende Band sich in ihrer Musik auf nordische Mythen bezieht; der umgekehrte Fall gilt ebenso, z.B. bei der schwedischen Band Amon Amarth, einer der bekanntesten Viking Metal-Bands, deren Name nicht aus der nordischen Mythologie, sondern aus J.R.R. Tolkiens Mittelerde-Romanen stammt (vgl. Foster 2002), obwohl ihre Songs nahezu ausschließlich von nordischen Mythen sowie von Wikingern handeln (vgl. Heesch 2012). Die der vorliegenden Studie zugrunde liegenden Beispiele wurden im Blick auf das übergreifende Thema nach folgenden Kriterien ausgewählt: Erstens sollte es um Bands aus Deutschland gehen, zweitens sollten sie aus Subgenres wie Black Metal und Pagan Metal stammen, in denen Mythenbezüge besonders gehäuft auftreten, und drittens sollten sie möglichst beispielhaft die Komplexität und Heterogenität sowohl der Zugänge zur Mythologie als auch des Verhältnisses zum Konstrukt »germanisch = deutsch $«$ veranschaulichen. Beim Aspekt der Komplexität geht es mir nicht um die Huldigung einer artifiziellen oder elaborierten Produktionsästhetik, sondern darum, den nicht eben einfachen Zusammenhängen einiger maßen gerecht zu werden. Dazu gehört unter anderem, das für Szene-

1 Umfangreichere Untersuchungen zum Nachleben der nordischen Mythen und Heldensagen im Allgemeinen sowie zur Mythenrezeption im Heavy Metal im Besonderen erfolgten im Rahmen des an der Goethe-Universität Frankfurt/Main angesiedelten interdisziplinären Forschungsprojekts »Edda-Rezeption«. 
Outsider möglicherweise unerwartete Expertentum zu erfassen, das hinter den Songs der Band Helrunar steht, oder aufzuzeigen, dass deutschtümelnde Darstellungen nicht nur mit simpler oder schlecht produzierter Musik daherkommen, sondern wohl oder übel auch mit Musik, die für ihre Qualität geschätzt werden kann, wie im Fall der Band Riger. Der Schwerpunkt meiner musikwissenschaftlichen Studie liegt auf der Untersuchung von Songs und Alben in ihrem Verhältnis zur Mythologie; doch es werden auch kulturelle Kontexte und größere ästhetische und historische Zusammenhänge untersucht. Im Folgenden liegt der Fokus in Abhängigkeit vom Beispiel einmal auf der Analyse von Musik und Lyrics, ein anderes Mal werden Interviewaussagen einbezogen, beim dritten Beispiel kommt eine Auswertung von Rezensionen aus einem Heavy Metal-Magazin hinzu.

\section{Zur > Germanisierung ‘ der nordischen Mythologie}

Die nordischen Mythen und Heldensagen, die in Deutschland häufig irreführend als >germanische Mythologie < aufgefasst werden, waren zunächst nur in Nordeuropa überliefert. Als wichtigste Quellen gelten zwei isländische Textsammlungen aus dem 13. Jahrhundert, die seit der Neuzeit beide als Edda bezeichnet werden: die Edda Snorri Sturlusons (Snorra Edda) und die so genannte Liederedda. Seit dem 18. Jahrhundert werden die darin enthaltenen Götter- und Heldengeschichten international rezipiert und in vielen Medien adaptiert (vgl. Clunies Ross/Lönnroth 1999; Schulz/Heesch 2009; Schulz 2011; Wawn 2005). Dabei wurden innen je nach Kontext diverse ideologische, nationalistische, religiöse und andere Bedeutungen zugeschrieben. In Deutschland begriff man diese Erzählungen schon bald nicht mehr nur als Teile der altnordischen Literatur, sondern als Zeugnisse einer gemeinsamen germanischen Kultur. Ein folgenreicher Impuls ging von Johann Gottfried Herder aus, der in seiner Konzeption vom »Volksgeist «, die auch in Nordund Osteuropa aufgegriffen wurde, das Volk als Träger des kulturellen Gedächtnisses und als neuen ästhetischen Orientierungspunkt für Gelehrsamkeit und Dichtung entdeckte (vgl. Steuer 2004: 395-398). In seinem Artikel »Iduna, oder der Apfel der Verjüngung « (1796) forderte Herder die deutschen Dichter dazu auf, sich in Ermanglung einer eigenen, deutschen Überlieferung mit der nordischen Mythologie auseinanderzusetzen. Die Grundlage dafür sah er in der Zugehörigkeit zum selben »Volksstamm«; gegenüber den in den Künsten dominierenden antiken Mythen glaubte Herder in den nordischen diejenigen zu erkennen, die »unserm nordischen Gefühl angemessener sind « (Herder 1998: 167 u. 169). 
Was sich in diesem Zusammenhang entwickelte, war das Konstrukt einer essentialistischen Kontinuität zwischen Germanen und Deutschen, das die Einverleibung der nordischen Mythologie als Teil der deutschen Volkskultur impliziert. Mittels dieses Konstrukts konnten die aus einer strikt an Quellen orientierten kulturhistorischen Sicht bestehenden Brüche in der Überlieferung der Mythen und Heldensagen übergangen werden. Solche Vorstellungen, die ich unter dem Begriff der essentialistischen Kontinuität zusammenfasse, können biologische, genealogische, ethnische, aber auch regionale Kontinuitätsvorstellungen beinhalten, wobei die »Region«, etwa >Germanien<, weniger als Kultur- denn als Naturraum konstruiert wird, der qua seiner natürlichen Beschaffenheit, z.B. Klima oder >Himmel<, eine bestimmte Kontinuität garantiere. ${ }^{2}$ Das wortwörtliche >Fortleben< der nordischen Mythologie und Heldensage wird demnach auf Naturvorgänge zurückgeführt bzw. auf das Leben des Menschen mit und in der Natur; der Bezug zur Mythologie wird in Vererbungsprozessen begründet, in der ethnischen und regionalen Verortung bzw. in einer Verankerung der Mythen im Volk.

Herders Impulse lösten unter Dichtern der deutschen Romantik wie Achim von Arnim und Clemens Brentano eine große Sammelleidenschaft für Volkslieder und Volksmärchen aus; unter wesentlicher Beteiligung von Jacob und Wilhelm Grimm entwickelte sich die Volkskunde. »Durch die Brüder Grimm und ihre Materialsammlungen bürgerte sich der romantische Gebrauch des Wortes >deutsch < im Sinne von >germanisch ein « (Steuer 2004: 395; Hervorhebungen im Original). Jacob Grimm setzte eine im Volk verankerte Kontinuität voraus, um in Märchen und Bräuchen Spuren vorchristlicher Kultur und Religion aufzufinden, und deutete von dieser Prämisse ausgehend in seiner Deutschen Mythologie (1. Aufl. 1835, Grimm 2001/2003), die nordische Mythologie als Bestandteil deutscher (Volks-)Kultur (vgl. Trümpy 1973: 5).

Im Kaiserreich erfuhr die Vorstellung einer genealogischen Kontinuität populäre Verbreitung durch historische Romane, wie Ein Kampf um Rom (1876) des Historikers Felix Dahn. Darin vermischt Dahn Geschichtliches, wie die Niederlage des ostgotischen Heeres in Italien im Jahr 553, mit dem Germanenmythos ${ }^{3}$ sowie mit Elementen aus altnordischer Literatur. Dahns

2 Der Begriff der Kontinuität verweist in der Geschichtswissenschaft generell auf »die Frage nach der Möglichkeit und den Voraussetzungen eines Zusammenhangs von Geschichte « (Herold 1976: 1038), die sich etwa, wenn nicht essentialistisch, im Blick auf kulturhistorische Entwicklungen beantworten lässt.

3 Als solcher ist ein »Herkunftsmythos « zu verstehen, wonach die Germanen den Ursprung einer eigenen kollektiven Identität darstellen; solcher Germanenmythos wurde bereits in der frühen Neuzeit, ausgehend von der Wiederent- 
Goten sind zugleich »Germanen « und »Deutsche « und kehren schließlich, indem sie von Wikingern gerettet werden, in die vermeintlich gemeinsame nordische Heimat Thule zurück. Die nordische Mythologie und Heldensage dienen auch hier der Konstruktion einer nordisch-germanisch-gotisch-deutschen Kontinuität. Einen zentralen, bis heute wirkenden Prägestempel erhielt das deutsche Germanenbild durch Wagners Operntetralogie Der Ring des Nibelungen (1876), die umfangreich aus der nordischen Mythologie schöpft, vermittelt unter anderem durch Grimms Zugang zu derselben (vgl. z.B. Jacobs 2009). Wagners Ring trug wesentlich dazu bei, sowohl die Gleichung »germanisch = deutsch « in der deutschen Kultur zu verfestigen als auch die sgermanischen Götter und Helden in nahezu allen verfügbaren Medien zu verarbeiten, wobei zu differenzieren ist zwischen Wagners Zugriff auf die Mythologie und den Zuschreibungen und Instrumentalisierungen, durch die er »erst im nachhinein zuständig für das Völkisch-Germanische « (Steuer 2004: 425) wurde. Auch wenn sich der Stellenwert dieser Vorstellungen im Verlauf des 20. Jahrhunderts stark gewandelt hat - am deutlichsten sichtbar wohl in ihrer nationalsozialistischen Rezeption sowie in der Distanzierung von derselben nach dem Ende des Zweiten Weltkriegs - bleiben die Grundelemente des Konstrukts einer germanisch-deutschen Kultur mit einer gemeinsamen >germanischen< Mythologie im Alltagsverständnis bis heute häufig unangefochten. Wie Heiko Steuer gezeigt hat, gilt das nicht nur für die Alltagskultur, sondern auch noch für Teile der Ur- und Frühgeschichtsforschung:

»Wie aktuell die Gleichung >germanisch-deutsch `noch heute ist und im Rahmen der geistesgeschichtlichen Situation unserer Gegenwart sich auswirkt, spiegeln nicht nur zum Beispiel die neuen Euro-Münzen Deutschlands, die weiterhin traditionell mit Eichenlaub geziert sind, sondern auch wissenschaftliche Diskussionsbeiträge der Ur- und Frühgeschichte zur ethnischen Deutung archäologischer Kultureinheiten« (Steuer 2004: 365).

An Wagners Mythenverarbeitung knüpfen übrigens einige Heavy Metal-Bands direkt an, die auch die weitere Auseinandersetzung mit der nordischen Mythologie in dem Genre angeregt haben, so die US-amerikanische Band Manowar und die schwedische Band Bathory, wobei in diesen Fällen der Aspekt des Deutschen keine bzw. kaum eine Rolle spielt; ${ }^{4}$ die historischen

deckung von Tacitus' Germania durch Humanisten, zur Abgrenzung von der römischen Kultur verwendet (vgl. Hirschi 2006).

4 Zum Wagnerbezug bei Manowar siehe Custodis 2009: 23-60. Der kreative Kopf von Bathory, Thomas »Quorthon « Forsberg, bezeichnete sich in den Liner Notes zum Album Blood On Ice (1996) als sfan of the life and works of Richard Wagner«, von dem er »more or less stole the legend of Siegfried and the aging and 
Bezüge im (internationalen) Heavy Metal sind komplex. Als Fazit aus dem kurzen historischen Überblick kann jedenfalls festgehalten werden, dass Bezugnahmen auf nordische Mythen innerhalb des deutschen Heavy Metal nicht nur auf Vorläufern in der Rockmusikgeschichte basieren, sondern an eine rund 200-jährige Geschichte der Mythenrezeption, die verwoben ist mit essentialistischen Vorstellungen einer Kontinuität von Germanen und Deutschen. Wie im Folgenden zu sehen sein wird, knüpfen Metal-Bands in ganz verschiedener Weise an diese kulturellen Vorbedingungen an.

\section{Bezüge zur nordischen Mythologie bei Helrunar}

Mythologische Bezüge - nicht nur nordische - finden sich zuhauf und in vielfältiger Form im Heavy Metal, speziell im Black Metal. Die grundsätzliche Frage, warum mythologische Elemente eine so wichtige Rolle im Metal spielen und welche Bedeutungen ihnen häufig zugeschrieben werden, kann im vorliegen Rahmen nicht umfassend erörtert werden (vgl. Campbell 2009; Heesch 2009; Höpflinger 2011; Walser 1993: 151-160). Festzuhalten ist, dass Mythisches zu den zentralen Heavy Metal-Themen gehört, aber in Abhängigkeit vom Subgenre und dem jeweiligen Individualstil der Bands sehr verschieden in der Musik verarbeitet wird. Als ein besonders anspruchsvolles Beispiel von Mythenadaption im Black Metal untersuche ich im Folgenden den Song »Unten und im Norden« aus dem Album Frostnacht (2005) der Band Helrunar. »Unten und im Norden« kombiniert ein Element aus der nordischen Mythologie mit einer Darstellung von Unterwelt, einem weiteren zentralen Metal-Thema. Helrunar spielen einen Black Metal-Stil mit folkloristischen Elementen, der stilistisch an den norwegischen Black Metal der 1990er Jahre anknüpft. Einige von dessen Vertretern, wie die Bands Enslaved oder Helheim, widmen sich (bis heute) ebenfalls schwerpunktmäßig Themen aus der nordischen Mythologie (vgl. Hagen 2011; Heesch 2011a; von Helden 2012; Mørk 2011). »Unten und im Norden« basiert, den formalen

dying gods of the Götterdämmerung, as well as the sword Notung. « Quorthon, der demnach bewusst »turned to the same books and legends « wie Wagner, regte mit Bathory, insbesondere mit den Alben Blood Fire Death (1988), Hammerheart (1990) und Twilight Of The Gods (1991) zahlreiche skandinavische und auch deutsche Bands dazu an, Elemente der nordischen Mythologie und Heldensage in ihrer Musik zu verarbeiten. Zur Geschichte der Band siehe Johannesson/Klingberg 2011. Ein besonders auffälliges Beispiel für eine Wagnerrezeption im deutschen Heavy Metal stellt die Band Grave Digger da, deren Album Rheingold (2003) ausdrücklich auf Wagners gleichnamiger Oper basiert. Allerdings würde es eine eigene Untersuchung erfordern, um zu klären, welche Rolle hierbei die deutsche Identität sowohl Wagners als auch der Band spielt. 
Genrekonventionen entsprechend, auf einer Reihung von Riffs (vgl. Elflein 2010: 50f. und passim), die durch rhythmische Modifikationen und Kontraste aufeinander aufbauen. Am Anfang wird zunächst durch tremolierende Gitarrenakkorde in verzerrtem Klang und das Einsetzen von Bass und Schlagzeug in triolischen Blast Beats ein Wall of Sound erzeugt, der sich durch periodisch wiederkehrende melodische Wendungen zum ersten Riff wandelt:

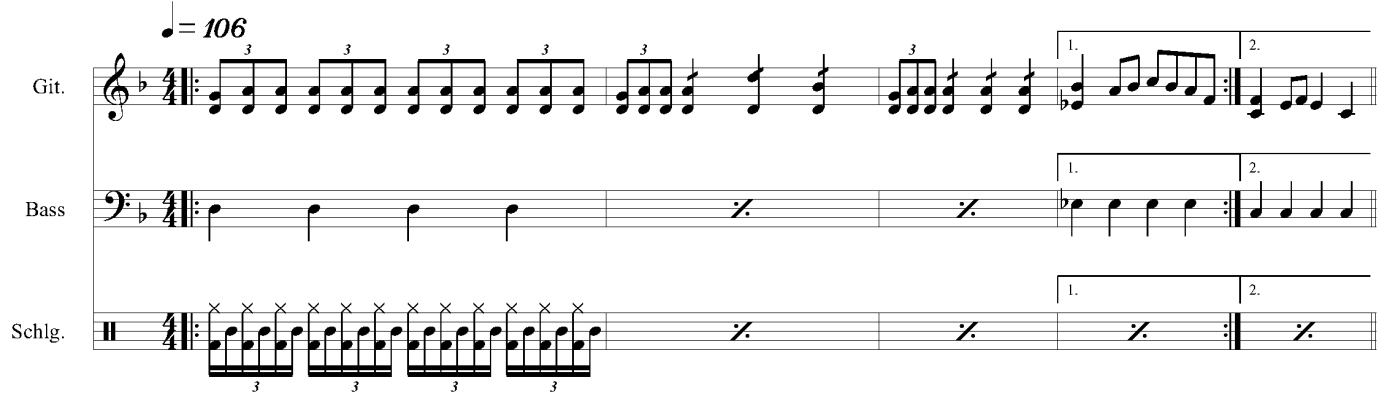

Notenbeispiel 1: Helrunar - »Unten und im Norden« (2005), Riff 1, schematische Transkription

Das anschließende Riff setzt sich davon unter anderem durch erhöhtes Tempo und einen binären (statt ternären) Blast Beat ab:

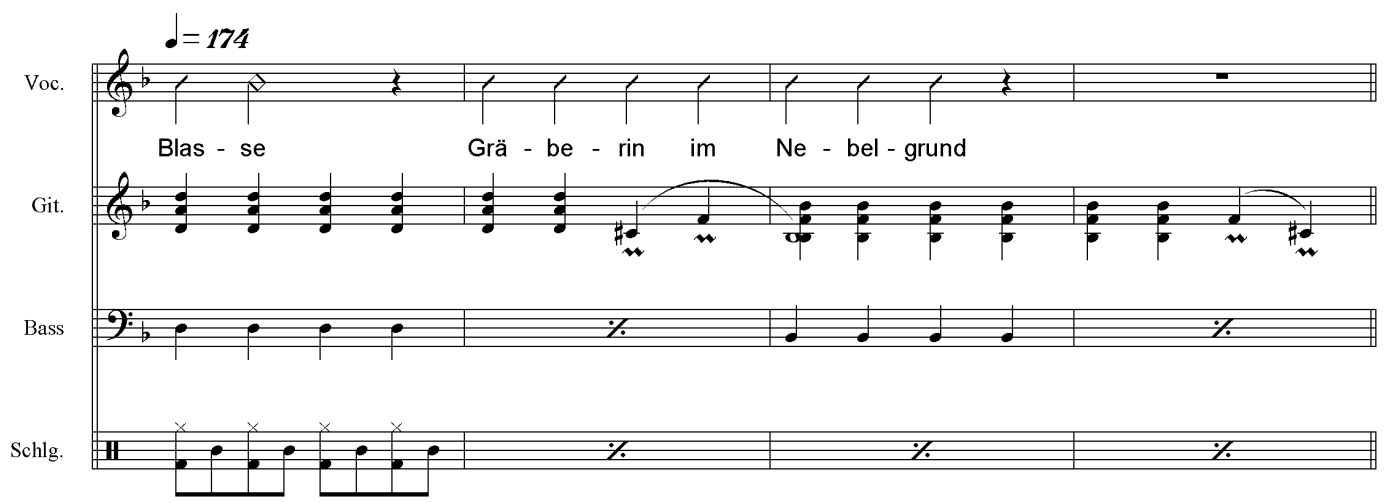

Notenbeispiel 2: Helrunar - »Unten und im Norden« (2005), Riff 2, schematische Transkription

Hinzu kommt der Sänger mit genretypischem krächzenden Screaming auf folgenden Text:

»Blasse Gräberin im Nebelgrund

Nebelspinne im Astgewirr

Zweige vor weißem Dezember

unten und im Norden «

(Helrunar: »Unten und im Norden«, 2005). 
Das, was sich »unten und im Norden« befindet, scheint eine weibliche Unterweltfigur zu sein, eine kühle Schauergestalt, die mit Tod und Winter zu tun hat. Mit dem nächsten Riff setzt ein schleppender, auf beinahe ein Drittel des vorangehenden und Tempos zurückgenommener >Mosh Part< (vgl. Elflein 2010: 283) ein, in den mithilfe elektronischer Produktionsmittel kurze Klangfenster eingebaut sind, die im Vordergrund des Gesamtmixes eine auf akustischer Gitarre gezupfte folkloristische Wendung hören lassen:

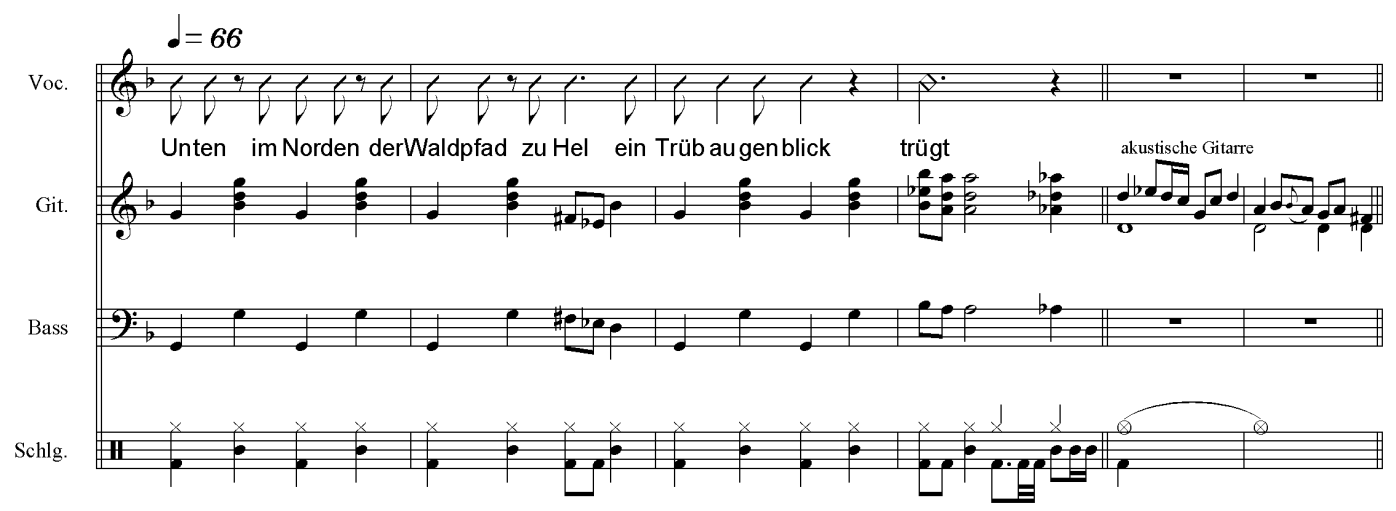

Notenbeispiel 3: Helrunar - »Unten und im Norden« (2005), Riff 3, schematische Transkription

Sodann erfolgt wieder ein Wechsel in rasendes Tempo; die Textur verdichtet sich durch Überlagerung von Tremoloakkorden und zweistimmigen Melodielinien in den E-Gitarren:

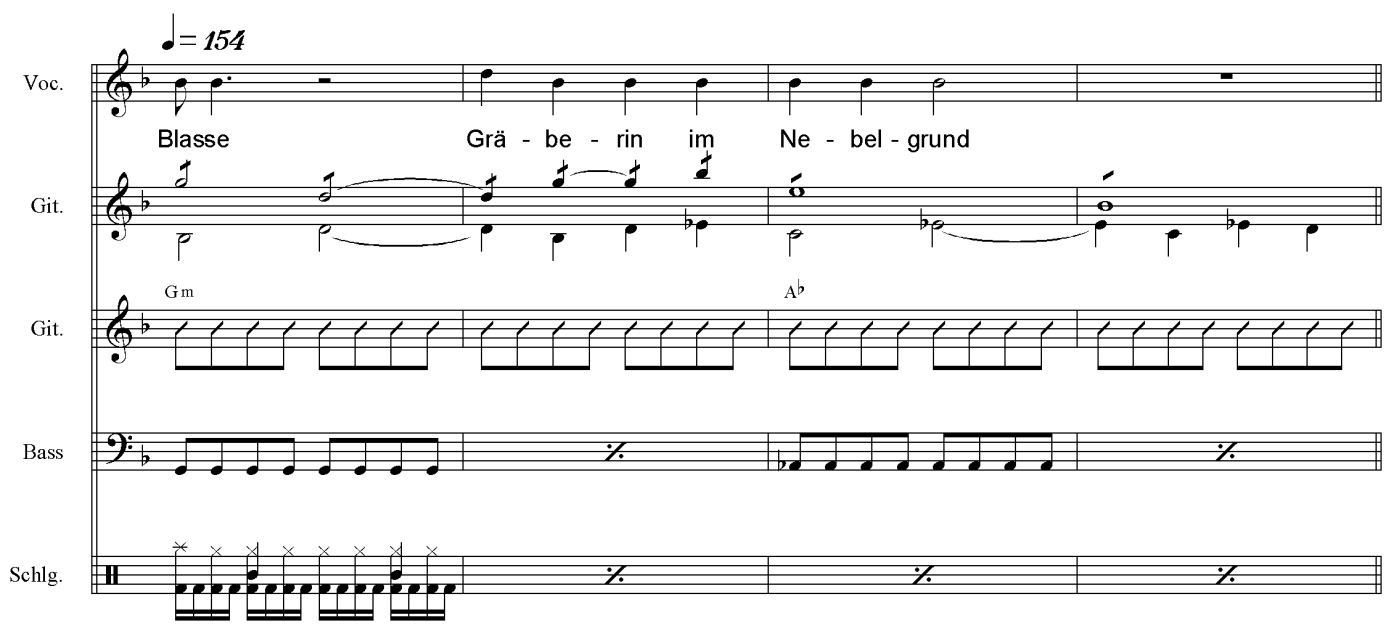

Notenbeispiel 4: Helrunar - »Unten und im Norden« (2005), Riff 4, schematische Transkription

Bis hierher mag die kurze Beschreibung genügen, um den durchaus komplexen Black Metal-Stil zu charakterisieren, der übrigens auch technisch und in 
Bezug auf die Produktion von hohem Anspruch zeugt. Auch der Songtext ist anspruchsvoll gestaltet und bedient sich einer betont lyrischen Sprache. Inhaltlich geht es in den vier Strophen - die oben zitierte erste Strophe wird als einzige am Ende wiederholt - um die Darstellung jener Unterweltgestalt, die erst in der letzten Strophe explizit als Hel identifiziert wird:

»Unten im Norden der Waldpfad zu Hel

ein Trübaugenblick trügt

ihr Haar ist ein Myzel

ihre Glieder ein Schlinggewächs...«

(Helrunar: »Unten und im Norden«, 2005).

Selten werden ganze Sätze formuliert; vielmehr reiht sich eine bildhafte Detailbeschreibung an die nächste, verknüpft durch lyrische Stilmittel wie Wort(teil)wiederholungen (»Nebelgrund « - »Nebelspinne «), Alliterationen (»Trübaugenblick trügt «) und einzelne Endreime (»Hel $«-\gg$ Myzel«). Außerdem finden sich gehäuft ungewohnte Begriffe und Komposita, neben dem mythologischen Namen Hel etwa die sich darauf reimende Bezeichnung eines Pilzgeflechts Myzel, des Weiteren »Nebelspinne«, »Dickichtgesicht « usw.

Der unterirdisch-dunkle, tödlich-kalte Charakter der Hel deckt sich mit deren Bedeutungsfeld in der nordischen Mythologie, wo sie allerdings für Zweierlei stehen kann: einerseits für das Totenreich und andererseits für dessen Personifizierung in Gestalt der Unterweltsgöttin, nach Snorri Sturloson als eines der monströsen Kinder, die der Gott Loki mit einer Riesin zeugte. Es ist jedoch nicht erst der Name Hel, der auf die nordische Totengöttin verweist, sondern bereits die mehrfach wiederholte Formel »unten (und) im Norden«, die dem Song auch seinen Namen gibt, denn damit wird eine Formulierung aus der Gylfaginning der Snorra Edda zitiert, die den Weg zur Herrscherin der Unterwelt beschreibt: »en niðr ok norðr liggr helvegr « (Gylfaginning 34 [49] in Edda Snorra Sturlusonar 1931: 66), auf Deutsch: »Und hinunter nach Norden verläuft der Helweg « (Die Edda des Snorri Sturluson 1997: 70).

Um die Formel im Song als Eddazitat zu erkennen, ist allerdings Expertenwissen notwendig, denn weder gehört sie zum allgemeinen Bildungsgut, noch ist sie in den Liner Notes zum Album als Zitat markiert. Zugleich finden sich innerhalb des Black Metal-Genres bereits Musikstücke, die die Formel aufgreifen, nämlich bei der bereits genannten Band Helheim im Titel von »Nidr Ok Nordr Liggr Helvegr « im Album Jormundgand (1995) sowie bei der ebenfalls norwegischen Band Burzum in den Liner Notes zu »Hermoðr á 
Helferð« (»Hermoðr auf der Fahrt zur Hel«) in Dauði Baldrs (1997). ${ }^{5}$ Jedoch handelt es sich in beiden Fällen um Instrumentalstücke; insofern kann man konstatieren, dass das Themenfeld um Hel und den Helweg samt der Formel »unten und im Norden« bereits vor Erscheinen von Helrunars gleichnamigem Song im Black Metal präsent ist, aber Helrunar daraus erstmals einen (vokalen) Song gestaltet haben. ${ }^{6}$

Die rockmusikalische Adaption eines Elements aus der nordischen Mythologie bei Helrunar verbindet also musikalische und literarische Originalität mit nichtmarkierten intertextuellen Bezügen zur mittelalterlichen Überlieferung der Mythologie sowie zur genreinternen Mythenrezeption im (Black) Metal. Dabei kann sich die Vielschichtigkeit der Bedeutungsebenen des Songs erst solchen Rezipierenden erschließen, die sich hörend und lesend näher mit nordischen Mythen auseinandersetzen. Helrunars Song ist insofern ein Beispiel für den hohen Anspruch, mit dem sich die Rezeption von nordischen Mythen in Teilen des Black Metal verbindet, sowie für die Verbreitung des Interesses an diesen Mythen, da offenbar ein entsprechend gebildetes Publikum vorausgesetzt wird. Dabei beschränkt sich dieser Zugang zu nordischen Mythen bei Weitem nicht auf diesen einen Song, sondern ließe sich an vielen weiteren Stücken von Helrunar untersuchen. Zum Beispiel vertonte die Band mit »Baldrs Draumar« (»Balders Träume«, auf Baldr ok Íss, 2007) den vollständigen altnordischen Text des gleichnamigen Eddalieds (Edda 1983: 277-279, deutsche Übersetzung in Die Götter- und Heldenlieder der Älteren Edda 2004: 181-185). Aufgrund der vokalen Gestaltung, in diesem Fall kein Screaming, sondern ein rezitierender Sprechgesang, ließe sich dieser Song durchaus mit so ambitionierten Projekten vergleichen wie den Edda-Vertonungen des Mittelalterensembles Sequentia (2002) oder eher noch, da es im Unterschied zu Sequentia nicht um die Rekonstruktion einer mittelalterlichen Klangwelt geht, mit den Adaptionen von (ins Norwegische

5 Burzum wird, zumindest im Rückblick, eine zentrale Position innerhalb der norwegischen Black Metal-Szene der frühen 1990er Jahre zugeschrieben. Notorische Berühmtheit erlangte die Band außerhalb der Szene vor allem aufgrund der kriminellen Taten und neonazistischen Äußerungen des Bandleaders Varg Vikernes, die in dem weit verbreiteten, apologetischen Buch Lords of Chaos (Moynihan/Søderlind 2003) ausführlich dargestellt werden (vgl. Heesch 2011a). Aufgrund dessen gehört Burzum zu den wohl meist kommentierten und kontrovers diskutierten Bands in der Heavy Metal-Forschung (vgl. z.B. Hagen 2011; Kahn-Harris 2007; Mørk 2011); jedoch steht eine nähere Auseinandersetzung mit ihrem musikalischen Stil meines Wissens noch aus.

6 Amon Amarth, eine der international bekanntesten Viking Metal-Bands, die stilistisch allerdings keinen Black Metal, sondern Death Metal spielt, veröffentlichte ebenfalls einen Song über den Weg zu Hel, jedoch erst 2006: »Hermod's Ride To Hel - Loke's Treachery Part I« auf dem Album With Oden On Our Side. 
übersetzten) Eddatexten durch den norwegischen Jazz-Pianisten und -Komponisten Ketil Bjørnstad (2001).

Bei »Unten und im Norden« handelt es sich um einen deutschen Song, in dem Elemente aus der altnordischen Überlieferung der nordischen Mythologie verarbeitet sind. Abgesehen von den deutschsprachigen Lyrics und der deutschen Herkunft der Band hat das Beispiel allerdings nichts mit der konstruierten Gleichsetzung »nordisch = deutsch « zu tun. In der Musik und ihren Paratexten (Albumcover etc.) geht es nirgendwo darum, die nordischen Aspekte als Teil einer deutschen Identität darzustellen; auch ist an keiner Stelle von etwas Germanischem die Rede. Das Beispiel steht insofern für das im Black Metal verbreitete Interesse an nordischer Mythologie, ohne jedoch ausdrücklich das Konstrukt einer Gleichsetzung des Deutschen mit dem Nordischen oder auch Germanischen zu untermauern.

\section{Germanen, Wikinger, Seeräuber und andere Barbaren. Bricolage-Praktiken bei Adorned Brood}

Themen aus dem Bereich der nordischen Mythologie oder der Geschichte der Wikinger erfreuen sich seit Mitte der 1990er Jahre großer Beliebtheit im Heavy Metal insgesamt; in den 2000er Jahren ist diese Beliebtheit zu einem Trend ausgewachsen, dem sich zahlreiche neue Bands anschlossen. Die 1993 in Grevenbroich gegründete Gruppe Adorned Brood gehört zu jenen deutschen Bands, die sich bereits relativ lange mit nordischer Mythologie auseinandersetzen. In der Genregenealogie gilt Adorned Brood als lang gediente Vertreterin des sogenannten Pagan Metal in Deutschland. Exemplarisch lässt sich die Verarbeitung nordischer Mythen an ihrem Song »A God Ruled Time« aus dem Album Asgard (2000) darstellen:

»In a time,

where magic spells and gods ruled.

In a time,

where Wotan held his hand above us.

Welcome in a time,

in which we can live as once.

Spread your wings...

Spread your wings and fly.

Life in the dark you open the wrong door.

Life in our Asgard we know it's our endevour [sic]«

(Adorned Brood: »A God Ruled Time«, 2000; zit. n. CD-Booklet). 
Noch stärker als in Helrunars »Unten und im Norden« wird der Black MetalStil hier kombiniert mit Folk-Elementen - eine Stilmischung, die in der Rockmusik seit Langem etabliert ist, in den frühen 1990er Jahren aber, angestoßen durch Bands wie die britischen Skyclad auch Einzug in so genannte Extreme Metal-Genres wie Thrash Metal und Black Metal gehalten hat. $\mathrm{Zu}$ den Black Metal-Elementen gehören in »A God Ruled Time « die schnellen Riffs, auch die reihende Songstruktur, die Sounds der verzerrten Gitarren und das helle Kreischen des Solosängers auf unbestimmter Tonhöhe; als Folk-Elemente werden damit kombiniert und kontrastiert: ternäre Rhythmen, melodische Männerchöre, der Einsatz von Flöten, die folkloristisch anmutende Linien spielen, sowie akustische Gitarren im Picking-Style. Dabei sind die Elemente dieser verschiedenen Stilbereiche so verflochten, dass nicht eines gegen das andere ausgespielt wird, sondern die Kontraste zu einem verbindenden Ganzen geführt werden: Rhythmisch und harmonisch, aber auch in Bezug auf die Hörbarkeit in der Abmischung fügen sich Flötenmelodien mit Metal-Rhythmen, Männerchöre mit Gitarrenriffs zusammen.

Aus semiotischer Sicht sind die verwendeten Folk-Elemente mit archaischer Kultur, Brauchtum und naturnaher Lebensweise assoziiert. Das ließe sich zum einen allein mit Bezug auf diesen einen Song nachweisen; allerdings handelt es sich zum anderen um Assoziationen, die bereits seit dem britischen und irischen Folk Rock der 1960er und 1970er Jahre, aber auch mit dessen späteren Nachfolgern im Black und Pagan Metal als Teil der Genrekonventionen verankert sind. Im Rahmen des vorliegenden Beitrags kann dieser Zusammenhang nur angedeutet werden; exemplarisch wäre etwa an Rockbands wie Jethro Tull, Thin Lizzy oder die erwähnte Folk Metal-Band Skyclad zu denken. Die Folk/Rock-Fusion korreliert dabei mit der Verarbeitung archaischer Themen in den Lyrics, ${ }^{7}$ in diesem Fall, bei Adorned Brood, ist es der Blick auf eine »God ruled time«, eine weit zurückliegende imaginierte Vorzeit, in welcher der Gott Wotan über ein Asgard herrschte, das belebt war von Zaubersprüchen, Kämpfen und männlichen Jagdgemeinschaften.

Beim Bezug auf mythologische Elemente fällt auf, dass Adorned Brood in diesem Song von Wotan sprechen, also einen südgermanischen Götternamen verwenden. An der Kombination von Wotan mit dem Götterwohnort Asgard (altnordisch Ásgarðr), der nur in der altnordischen Überlieferung Erwähnung

7 Zur Folk/Rock-Fusion in England siehe Sweers 2005, zum Fusion-Begriff in diesem Zusammenhang auch Sweers 2002; zum britischen Progressive Rock, seiner Verwurzelung im Folk Revival und seiner Vorliebe für Archaisches siehe Macan 1997. 
findet, zeigt sich, wie die nordische Mythologie als eine germanische Mythologie dargestellt wird. Diese Auffassung von einer germanischen Mythologie erweist sich im Blick auf Adorned Broods musikalisches CEuvre als symptomatisch; zudem spiegelt sich darin die Fortsetzung der Gleichung "nordisch = germanisch $«$ als $»$ nordisch = germanisch = deutsch $«$. So erscheinen etwa im Debütalbum Hiltia (1996) die Vertonung von Auszügen aus dem altnordischen Eddalied Hávamál in deutscher Übersetzung (»Die Rede des Erhabenen $«$ ) sowie ein Kampflied mit dem Titel »Furor Teutonicus«. Es wäre allerdings verfehlt, Adorned Broods Musik als bewusste Untermauerung des Konstrukts jener Gleichsetzung von Nordischem, Germanischem und Deutschem oder gar als nationalistische Propaganda zu deuten, zumal man vergeblich nach Aussagen sucht, die explizit das Deutsche betonen. In erster Linie wird hier - durchaus unreflektiert - an das verbreitete Klischee "germanisch = deutsch« angeknüpft. Wie eingangs dargestellt, wird das Konstrukt einer nordisch-germanisch-deutschen Identität außerhalb von Fachdiskursen selten infrage gestellt. Insofern zeugt Adorned Broods Adaption des Konstrukts weniger von einem reflektierten Vorgehen als von einem Rückgriff auf verbreitete Germanenbilder, der - anders als etwa bei Helrunar - ohne elaboriertes Quellenstudium auskommt.

Aufschlussreich ist in diesem Fall, wie die Musiker von Adorned Brood ihren Zugang zur nordischen Mythologie beschreiben. Am Rand des sechsten Ragnarök-Festivals in Lichtenfels am 17. und 18. April 2009 hatte ich gemeinsam mit der Skandinavistin Imke von Helden Gelegenheit zu einem Interview mit zwei Mitgliedern der Band, dem Sänger und Texter Markus Frost und dem Gitarristen Mirko Klier (der inzwischen nicht mehr bei Adorned Brood spielt):

Imke von Helden (IvH): »Zu den Konzepten hinter den Liedern, den Lyrics, wer schreibt die für euch?«

Frost: »Für die neue Scheibe [Noor (2008)] hab ich die geschrieben. [...] Die geht ja auch, die geht komplett über Seefahrer - fast, nicht jedes Lied. Zum Beispiel >Adorned Brood < beschreibt eigentlich unseren Namen, das Lied, und der Rest ist eigentlich über die Seefahrer.«

IvH: »Bei den alten Sachen habt ihr ja noch mehr nordische Mythologie. Wie ist das gekommen?«

Frost: »Schon immer, habe ich mich schon immer für interessiert. Nordische Mythologie, Wikinger, Germanen.«

IvH: »Also alles querbeet?«

Frost: »Ja. [...] Also, jetzt nicht nur Wikinger oder Germanen — ist ja alles das Gleiche.« 
Mirko Klier: »Seeräuber...«

Frost: »Seeräuber, genau. Wikinger heißt ja eigentlich Seeräuber.«

Demnach scheint hinter der Musik von Adorned Brood ein Interesse für solche Themen zu stehen, die stereotypen Vorstellungen nach mit archaischer Kultur und Religion, heroisch-kämpferischen, männlichen Outlaws und einer nicht näher spezifizierten germanisch-nordischen Ethnizität assoziiert werden. Dabei geht es eher um die Mischung als um eine Differenzierung der diversen Themenbereiche, schließlich »ist ja alles das Gleiche«.

Nach seinen Quellen gefragt, hob Frost im Interview zwei Dinge hervor, nämlich eine Überblicksdarstellung zu den Germanen: »ein supergeiles Buch [...], das heißt nur > Die Germanen < und da steht halt alles drin, alles, wat de wissen willst«. Um welches der zahlreichen Bücher mit diesem Titel es sich hierbei handelt, konnte ich nicht ermitteln. Als zweite wichtige Quelle nannte Frost den Zeichentrickfilm Walhalla, eine Comicadaption des dänischen Autors Peter Madsen, die 1986 auf Dänisch (Valhalla) und 1987 in deutschsprachiger Fassung erschien. Der Film handelt von zwei Kindern, Tjelfe und Roskva, die in vormoderner Zeit auf einem skandinavischen Bauernhof aufwachsen, bis sie den Göttern Thor und Loki begegnen und mit ihnen und dem Riesenkind Quark eine Reihe von Abenteuern bestehen, die auf verschiedenen Erzählungen aus der nordischen Mythologie basieren.

Unter jenen Heavy Metal-Musikern, denen es bei ihrer Auseinandersetzung mit Mythen im Unterschied zu Vertretern wie Helrunar weniger auf eine genaue Orientierung an historischen Quellen ankommt, kann Frosts Zugang zu den Mythen vermutlich als typisch gelten: Zum einen beruft er sich auf leicht zugängliche Literatur, die in erster Linie einen schnellen Überblick bietet; zum anderen greift er auf bestehende Mythenadaptionen der populären Kultur zurück. Insbesondere liegen Mythen- und Fantasyadaption häufig eng beieinander oder vermischen sich. ${ }^{8}$ So findet sich auch bei Adorned Brood, wie bei vielen Metal-Bands, ein Bezug auf J.R.R. Tolkiens Mittelerde-Romane, nämlich in der Verwendung des Namens Narog, in Tolkiens Silmarillion der Name eines Flusses (vgl. Foster 2002), im Song »Black Beasts« auf Asgard.

Ein solcher Bezug auf die nordische Mythologie lässt sich als eine Bricolage verstehen, indem mythische Elemente aus diversen Quellen entnommen, frei kombiniert und mit anderen Quellen, etwa aus dem FantasyBereich, gemischt und in neue Sinnzusammenhänge gestellt werden. ${ }^{9}$ Ganz

8 Zu Manowar und ihrer Zusammenarbeit mit dem Fantasy-Autor Wolfgang Hohlbein siehe Heesch 2011b.

9 Der von Claude Lévi-Strauss geprägte Begriff der Bricolage hat sich vor allem durch die Studien von Dick Hebdige zur Beschreibung subkultureller Praktiken 
in diesem Sinn enthält das jüngere Album Noor (2008) neben einem Song über ein nordisch-mythologisches Thema, »Under Yggdrasill«, unter anderem eine Neuvertonung des aus sogenannten Volksliedsammlungen bekannten Liedes »Wir lieben die Stürme« (vgl. Widmaier 2012), das hier unter dem Titel »Am Grunde des Meeres« erscheint, des Weiteren eine Rockversion des Shanties »What Shall We Do With The Drunken Sailor .

Angesichts dieses bricolageartigen Umgangs relativiert sich die ideologische Aussagekraft der Gleichsetzung von Nordischem mit Germanischem und Deutschem. Zweifellos greifen Adorned Brood unkritisch auf solche stereotypen Gleichsetzungen zurück; insofern mag ihre Musik durchaus zum Fortbestehen stereotyper Vorstellungen von germanischer Mythologie als archaischer Religion der Deutschen beitragen. Aus einer historisch-kritischen Sicht sowohl auf die deutsche Geschichte als auch auf die Geschichte germanischer Stämme und die Überlieferung der nordischen Mythologie muss sich Adorned Broods Darstellung als haltlos erweisen. Indes wird aus der Bricolage ersichtlich, dass es hier mitnichten um den Versuch einer historischen Rekonstruktion geht, sondern bewusst mit Stereotypen und Fiktionen gespielt wird. Das Nordisch-Germanische und auch das Deutsche sind hier Elemente einer Fantasy-Kultur. Das macht solche Bezüge zurecht nicht unangreifbar gegenüber Kritik an essentialistischen oder regressiven Implikationen, wie anhand der umfangreichen wissenschaftlich-kritischen Auseinandersetzung mit solchen Aspekten in der Fantasy-Literatur zu sehen ist. ${ }^{10}$ Allerdings steht hier offenbar der spielerische, unterhaltende Umgang mit mythischen Bezügen im Vordergrund.

\section{»Sippenheil«. Essentialistische Tendenzen bei Riger}

Die 1996 in Frankfurt/Oder gegründete Band Riger ist ein Beispiel dafür, wie das Konstrukt einer germanischen Mythologie in ein Zeichengewebe eingebunden sein kann, das deutliche politische Implikationen aufweist. Dabei ist eine genaue Interpretation notwendig, um der Komplexität des Beispiels gerecht zu werden, denn man hat es hier nicht mit dumpfem Neonazirock zu tun: Zum einen erscheinen die politischen Aspekte nicht in Form plakativer Parolen oder offener Verwendung verfassungsfeindlicher Symbole,

etabliert (vgl. Winter 2001: 121-124); in ähnlicher Weise verwendet ihn bereits Walser (1993: 151-160), um die Mischung mythologischer, literarischer und historischer Elemente bei der Heavy Metal-Band Iron Maiden zu charakterisieren.

10 Siehe exemplarisch Schnurbein (2007). 
sondern als teils verschleierte bzw. komplexe Bezüge; zum anderen ist die Musik von solcher Qualität, dass sie Black Metal-Fans auch ohne Einverständnis mit den Inhalten ästhetisch anzusprechen vermag. In Metal-Magazinen wurden Riger anfangs als eine der bis dato selten guten Black Metal-Bands aus Deutschland gewürdigt. So begrüßte Andreas Stappert Rigers Debütalbum Der Wanderer (1998) in Rock Hard mit den Worten:

»Warum in die Ferne (respektive gen Skandinavien) schweifen, wenn der gute Black Metal so nahe, also quasi vor der eigenen Haustür abschimmelt? Nach Falkenbach, Atanatos und Agathodaimon drängt nun mit RIGER aus Frankfurt/Oder eine weitere gute Band aus unserer zuweilen recht seltsamen Republik ans Licht der schwarzmetallischen Öffentlichkeit« (Stappert 1998).

Rigers Songs fallen hinsichtlich Komposition, spielerischer Umsetzung und Produktion als qualitativ gut auf und sind teils komplex, so das achteinhalbminütige Stück »Baum«, das Stappert in seiner Rezension hervorhebt. Auch das zweite Album, Hamingja (1999), wurde in Rock Hard für die hohe Qualität und Komplexität der Musik in Black Metal-typischer Bildersprache gelobt:

»)Hamingja< ist ein schillerndes Stück Schwarzkunst, eine sinnige Einheit aus aggressiver, böser Kraft und schwebender Spiritualität. RIGER sind gleichzeitig talentierte Lyriker und Brechstangenschwinger. [...] >Hamingja< wirkt zu keinem Zeitpunkt plakativ und besticht dennoch durch Entschlossenheit. Wie soll man das nennen? Intelligenzler-Black Metal? Ich weiß nicht. Bleiben wir lieber bei dem Wort >Musik« (Mühlmann 2000).

In einem zurückhaltenden Postscriptum äußert Wolf-Rüdiger Mühlmann eine hier noch sehr vorsichtige, durch Anspielungen angedeutete Kritik an der Cover-Gestaltung des Albums: »Das zweideutige Coverartwork allerdings ist so überflüssig wie ein Kropf, denn eigentlich sollte es die Band nicht nötig haben, sich bei thüringischen Idioten einzuschleimen. « Mit jenen »thüringischen Idioten « dürften insbesondere Mitglieder und gleichgesinntes Umfeld der neonazistischen Band Absurd aus Sondershausen gemeint sein, die ihre thüringische Identität und ihre (in stilistischer Hinsicht fragwürdige) Zugehörigkeit zum Black Metal plakativ betonten und sich »zu einem der Kristallisationspunkte des extrem rechten Underground« (Dornbusch/Killguss 2005: 147) entwickelten; daneben haben sich gerade in Thüringen weitere Black Metal-Bands durch rechtsextreme Äußerungen hervorgetan. ${ }^{11}$ Allerdings hält

11 So Dornbusch/Killguss 2005: 171-177; allerdings bieten deren Beobachtungen, die in vielen Fällen durchaus gründlich und fundiert sind, keinen Überblick über die deutsche bzw. hier speziell die thüringische Black Metal-Szene insgesamt, denn es geht ihnen vor allem um eine kritische Bestandsaufnahme rechtsextremer Tendenzen aus einer dezidiert antifaschistischen Position. 
sich Mühlmann mit seiner Beschreibung der Coverillustration als »zweideutig« meines Erachtens allzu sehr zurück: Dass die Darstellung von je vier Armen und Drachenköpfen die Form übereinander gelagerter Hakenkreuze ergibt, mag zwar nicht hinreichend plakativ im Sinn der Verwendung verfassungsfeindlicher Zeichen sein, ist aber unzweifelhaft zu erkennen. ${ }^{12}$

Rigers Selbstinszenierung und Musik zeugen von einer intensiven Auseinandersetzung mit nordischer Mythologie. So ist bereits der Name der Band dem Eddalied Rígspula (Merkgedicht von Rig) entlehnt, in dem ein Gott unter dem Namen Rígr auftritt (vgl. Edda 1983; Die Götter- und Heldenlieder der $\ddot{A}$ lteren Edda 2004). Hinter dem Titel des Albums Hamingja verbirgt sich ein altnordischer Begriff, der in isländischen Sagas sowie im Eddalied Vafprúðnismál (Wafthrudnirlied) 48-49 belegt ist und im Deutschen meist als »Glückskraft« oder »Schutzgeist« gedeutet wird (vgl. Beck 1999). Riger stellen ihre Interpretation dieser Kraft im Titelsong des Albums dar:

»Der Väter, der Ahnen und unser Ruhm

soll in jenem Schicksal ruhn.

Hamingja.

Wir rasen durch das Eichenlaub.

Wir sind Germaniens Scharen.

Und jede Kraft, die uns aufhält,

soll unsern Zorn erfahren.

Hamingja.

Die eherne Kette zwischen Mann und Mann

verbindet uns in Seel und Tun.

Der kostbare Wert, der in uns verrann,

wird nimmer in Stille ruhn «

(Riger: »Hamingja«, 1999).

Die lyrische kollektive Instanz, die sich hier als kriegerische germanische Männerschar ausgibt, beruft sich mit dem Begriff Hamingja auf eine schicksalhafte, kraftverleihende Verbindung zu den Ahnen. Vermutlich knüpfen Riger dabei an die in Fachkreisen für ihre Eigenständigkeit bekannte Hamingja-Interpretation des dänischen Religionswissenschaftlers Vilhelm Grønbech (eingedeutscht Wilhelm Grönbech) an, für den die Hamingja »die Verkörperung des Sippenheils« (Beck 1999) bedeutet. Grønbechs weit verbreitete Darstellung germanischer Kultur, die zuerst 1909-1912 unter dem Titel Vor folkeat i oldtiden (»Unser Volksgeschlecht in alter Zeit«) und 1931 in einer erweiterten englischen Fassung The Culture of the Teutons veröffentlicht

12 Die Abbildung ist nach heutigem Stand (März 2014) mittels einer simplen Internetrecherche nach »Riger Hamingja« leicht auf zahlreichen Websites zu finden. 
worden war, erschien in Deutschland unter dem Titel Kultur und Religion der Germanen erstmals 1937, in fünfter Auflage 1954 und bis heute in 13 Auflagen sowie mehreren Nachdrucken. Gleich in den ersten Sätzen der Einleitung macht Grønbech deutlich, dass Skandinavier, Engländer und Deutsche nach seinem Verständnis einer gemeinsamen Kultur entstammten:

»Unter dem Namen Germanen fassen wir den Volksstamm zusammen, von dem die Skandinavier, Deutschen und Engländer Abzweigungen sind. Der Name selbst ist vielleicht von außen gekommen, uns von Fremden gegeben worden. [...] Die Römer, die zwischen den Bewohnern Galliens und ihren östlichen Nachbarn zu unterscheiden wußten, nannten diese letzteren Germanen; mit Recht wurde dadurch die enge Verwandtschaft hervorgehoben, die von den frühesten Zeiten an die Bewohner der nördlichen und südlichen Ostseeländer mit den Flußufer- und Waldvölkern in Norddeutschland verband, eine Verwandtschaft, die sich nicht nur in der Sprache kundgibt, sondern noch weit mehr in der Kultur bis in ihre innersten Verzweigungen hinein « (Grönbech 1954: 17).

Die englische und deutsche Übersetzung seines Buchs und die Verwendung der Begriffe »Teutons « und »Germanen« in den Titeln implizieren also keine Vereinnahmungen einer skandinavischen Perspektive, sondern entsprechen Grønbechs Auffassung. Diese kann aus heutiger Sicht trotz ihrer ausgewogenen und sachlichen, dem Stand ihrer Zeit entsprechenden Darstellung nicht mehr für ein historisch differenziertes Bild von den germanischen Stämmen und ihren Zeitgenossen stehen; sie kann indes geeignet sein, stereotype Vorstellungen entsprechend der Gleichsetzung "nordisch = germanisch = deutsch « zu bestärken. ${ }^{13}$ Zur Hamingja schreibt Grønbech:

»Das Geschlecht ist ursprünglich eine Hamingja, und als Seele wird es den neuen Verwandten einverleibt. Die Personen sind nur Repräsentanten der Hamingja, und ihre Macht besteht darin, daß sie imstande waren, das Leben, das, reich an hervorragenden Charakterzügen, durch ihre Vorfahren flutete, wiederzubeleben. Die Hamingja ist immer etwas Gegenwärtiges, und die Vergangenheit ist nur wirklich, sofern ihr Geschick in einer Reihe von vergangenen Generationen immer wieder erneuert worden ist« (Grönbech 1954: 387).

Im Bezug auf die grønbechsche Deutung der Hamingja perpetuieren Riger ein Konzept von essentialistischer Kontinuität zu germanischen Vorfahren. Dabei zielt Grønbech nicht auf so etwas wie »reine« Vererbung, sondern deutet die Hamingja als eine flexible Größe, die sich in bestimmten Situa-

13 In der Altertumswissenschaft um 1900 fühlten sich auch die skandinavischen Forscher »als Germanen, die ihren bronzezeitlichen Vorfahren unmittelbar verbunden waren « (Steuer 2004: 385); allerdings war die Verknüpfung mit rassistischen Vorstellungen in Deutschland stärker verbreitet (ebd.: 386). 
tionen »zu der Dimension eines Stammes oder sogar eines Volkes anschwellen (Grönbech 1954: 390) konnte: »In Kriegszeiten ging das Heil seines [des Königs] Gefolges in ihm auf, und so wurden seine Götter und Vorfahren die Götter und Vorfahren des ganzen Volkes« (ebd.). Im Rahmen der vorliegenden Studie konnte nicht näher untersucht werden, wie detailliert die Musiker von Riger sich mit Grønbech auseinander gesetzt haben. Selbst wenn sie diese Differenzierung des Erbschaftsverhältnisses der Hamingja zur Kenntnis genommen haben sollten, ließe sich schwerlich beurteilen, ob dies die Vorstellung einer Verwandtschaft mit >den Germanen< eher bestärkt oder nicht. Jedenfalls dürfte die Idee von der Hamingja als einem germanischen Sippenheil mehr oder weniger direkt von Grønbech übernommen worden sein. Dabei wird klar, dass Riger in Songs wie »Hamingja« Vorstellungen von essentialistischer Kontinuität zwischen heutigen Deutschen und vorzeitlichen Germanen (im stereotypen Sinn kriegerischer Männerbünde) darstellen. Der Bezug zur nordischen Mythologie geht hier einher mit einer Affirmation gegenüber einer essentialistischen Gleichsetzung von Deutschen und Germanen. Das wird auch deutlich im Vergleich mit den Mythologiebezügen bei Bands wie Helrunar, die sehr literatur- und geschichtsorientiert arbeiten, oder Adorned Brood, die eher bricolageartig Mythisches mit populären Klischeebildern kombinieren.

\section{Fazit und Ausblick}

Die Verarbeitung von (nicht nur nordischen) Mythen und Referenzen auf archaische Kulturen ist im Heavy Metal weit verbreitet. Ein Teil der Musikerinnen, Musiker und Fans setzt sich intensiv mit entsprechenden historischen Darstellungen und literarischen Zeugnissen auseinander; manche gehen diesem Interesse sogar im Rahmen eines Studiums der Skandinavistik oder anderen Fächern mit thematischen Berührungspunkten nach; für manche verbindet sich das Interesse an Wikingern oder anderen mittelalterlichen Kulturen mit einem Interesse an Fantasy-Literatur und Filmen oder an Rollenspielen, etwa auch dem mehr oder weniger streng an historischen Modellen orientierten Nachstellen alter Kampfpraktiken. Metal-Fantum impliziert häufig ein gewisses Expertentum nicht nur im Hinblick auf die Musik, sondern auch in Bezug auf Themen. Dabei ist im Blick auf die Heavy MetalKultur insgesamt deren Vielfältigkeit und Heterogenität zu beachten, auch wenn dies im vorliegenden Rahmen nicht en detail diskutiert werden kann: Neben mythologischen werden viele weitere Themen behandelt, wobei dies auch je nach Subgenre variiert. Genres wie Pagan Metal und Viking Metal 
definieren sich gerade über Themen wie archaische Religion, Kultur und Kriege; auch in Black Metal und Power Metal werden solche Themen gehäuft verarbeitet.

Trotz der teils intensiven Auseinandersetzung mit Themen und historischen Hintergründen findet eine kritische Diskussion der ideologischen Implikationen in der Regel allenfalls ansatzweise statt. Im Fall Riger war zu beobachten, dass das Rock Hard-Magazin hier, wenn auch nach einigem Zögern, eine kritische Diskussion angestoßen und sich schließlich in eindeutiger Distanznahme positioniert hat. ${ }^{14}$ Jüngere Publikationen von Riger wurden im Rock Hard, das sich betont unabhängig gibt, vorerst nicht mehr besprochen, auch nicht in dem vom Springer-Verlag herausgegebenen Metal Hammer. ${ }^{15}$ Allerdings werden solche Magazine nur von einem Teil der Fans gelesen; abgesehen davon hat das insgesamt weniger kritische Magazin Legacy sich dem Rezensionsboykott nicht angeschlossen. ${ }^{16}$ Es muss konstatiert werden, dass eine kritische Auseinandersetzung mit ideologischen Tendenzen im Heavy Metal insgesamt zu kurz kommt. Der Soziologe Keith KahnHarris (2007: 141-156) hat das häufige Ausbleiben von Kritik in der Szene als »reflexive anti-reflexivity « beschrieben. Demnach werden Themen und Inhalte durchaus auf hohem Diskussionsniveau reflektiert, allerdings stoppt die Reflexion regelmäßig an bestimmten Punkten, gerade in Bezug auf politische Aspekte - hier kippt der szeneinterne Diskurs in eine Antihaltung zur Reflexion.

Es stellt sich aber auch die Frage, wo Kritik ansetzen sollte. Eindeutig rechtsextreme Positionierungen finden sich nur in Nischen der Metal-Kultur und werden von den meisten Fans abgelehnt. Anders sieht es mit affirmativen Darstellungen der vermeintlich eigenen Kultur und Geschichte aus. Dass zahlreiche Bands, insbesondere im Black Metal, Pagan Metal und Viking

14 So geht Mühlmann (2002) in seiner Rezension des folgenden Riger-Albums Des Blutes Stimme (2002) auf kritische Distanz zu den Lyrics und sieht sie im Konflikt zur durchaus ansprechenden Musik: "Störend wirken auf mich die deutschsprachigen Blut-, Heimat- und Schlachtentexte, denn Songtitel wie >Teutonenzorn< sind meines Erachtens einfach nur dämlich. Irgendwie hört sich der Lyrikeintopf an wie die Metalversion von Oktoberfest meets NPD-Versammlung. Und das wiederum schmälert den Gesamteindruck erheblich. «Im Folgeheft von Rock Hard erscheint unter der Rubrik »Krach von der Basis« ein mit »Riger. Provokation oder Missverständnis? « betiteltes Interview mit Rigers Gitarrist Peter Patzelt, in dem unter anderem kritisch auf die Hakenkreuzdarstellung des Hamingja-Covers eingegangen wird (Mühlmann 2003).

15 Nach Recherchen in den Review-Archiven von Rock Hard und Metal Hammer, die von den Verlagen kostenpflichtig angeboten werden; Stand vom März 2012.

16 So findet sich in der Rezension von Rigers Album Streyf im Legacy ChroniclesSonderheft Pagan Fire 2 (Eck 2008/2009) keine Spur von kritischer Auseinandersetzung. 
Metal, sich auf sihre < vermeintlich alte Herkunft berufen, dabei auch von »Ahnen« oder - allgemeiner, aber ebenfalls eine Form von Abstammung implizierend - von »Wurzeln « singen bzw. sprechen, zeugt von der relativ unangefochtenen Verbreitung essentialistischer Vorstellungen von ethnischer bzw. nationaler Kultur. Solche Ideen werden aus der Innensicht kaum problematisiert. Im Blick auf die hier im Fokus stehende Frage nach essentialistischen Vorstellungen von deutscher = germanischer Identität muss man indes auch in Betracht ziehen, in welchem Zusammenhang überhaupt von solcher Identität gesprochen wird. Nur ein Teil der Bands, die nordische Mythen verarbeiten, deuten diese als germanische oder gar als deutsche, zum Beispiel Riger. Im Fall von Helrunar bleibt eine solche Deutung völlig aus; bei Adorned Brood geschieht sie eher implizit oder in Interviewaussagen, während die Musik durch die Bricolage einen eher spielerischen, deutungsoffenen Zugang zum > Germanischen< zeigt. Vereinnahmungen der nordischen Mythologie als germanisch-deutsche im Sinn essentialistischer bzw. nationalistischer Vorstellungen finden sich also nur in einem Teil der Mythenbezüge und das in je verschiedener Ausprägung. Hier gilt es, im konkreten Einzelfall genau hinzuschauen.

Beim Thema Mythenrezeption muss man schließlich auch im Blick behalten, dass Heavy Metal zwar zu einem Teil in lokal gebundenen Szenen praktiziert wird, aber im großen Ganzen eine internationale Kultur ist. Manche Musikerinnen und Musiker singen in anderen Sprachen und über andere Mythen, die mit ihrer Herkunft nichts zu tun haben; umgekehrt können sich Fans für Musik über >fremde -Mythen begeistern. Durch transnationalen und transkulturellen Austausch werden essentialistische Zugänge zu Mythen relativiert. Was man dabei meiner Ansicht nach kritisch beobachten muss, sind Tendenzen, spezifische Mythologien heutigen Ethnien, Nationen oder Regionen im Sinn einer ererbten kulturellen Identität zuzuschreiben, wonach zum Beispiel heutige Skandinavier als >natürliche Erben heidnischer Wikinger und ihrer Mythologie gelten, heutige Iren sich hingegen eher an Keltischem zu orientieren hätten usw. Es muss klar gesagt werden, dass eine solche Sichtweise zum Ethnopluralismus tendiert, also zu der Vorstellung, dass sich kulturelle Vielfalt auf separate Ethnien und Regionen verteilt, was letztlich einem essentialistischen Kulturverständnis Vorschub leistet. Sofern die Internationalität des Heavy Metal insgesamt allerdings dazu beiträgt, essentialistische Vorstellungen von Nation und Ethnie zu unterwandern, kann sie (hoffentlich) auch Vorstellungen vom >typisch Deutschen< relativieren. 


\section{Literatur}

Beck, Heinrich (1999). »Hamingja.«In: Reallexikon der Germanischen Altertumskunde. Bd. 13. Hg. v. Heinrich Beck, Dieter Geuenich und Heiko Steuer. Berlin: de Gruyter (2., vollst. neu bearb. u. stark erw. Aufl.).

Bénard, Nicolas (2004). "De la légende viking au Hard-Rock. Les références culturelles du Métal nordique. «In: Nordiques 5 (été automne), S. 55-68.

Campbell, lain (2009). »From Achilles to Alexander. The Classical World and the World of Metal. « In: Heavy Metal Music in Britain. Hg. v. Gerd Bayer. Farnham, Burlington: Ashgate, S. 111-124.

Clunies Ross, Margareth / Lönnroth, Lars (1999). "The Norse Muse. Report from an International Research Project. «In: alvíssmál 9, S. 3-28.

Custodis, Michael (2009). Klassische Musik heute. Eine Spurensuche in der Rockmusik. Bielefeld: transcript.

Dahn, Felix (1876). Ein Kampf um Rom. Historischer Roman. Berlin: Deutsche BuchGemeinschaft (Sonderdruck).

Die Edda des Snorri Sturluson (1997). Ausgewählt, übersetzt und kommentiert von Arnulf Krause. Stuttgart: Reclam.

Die Götter- und Heldenlieder der Älteren Edda (2004). Übersetzt, kommentiert und hg. v. Arnulf Krause. Stuttgart: Reclam.

Dornbusch, Christian / Killguss, Hans-Peter (2007). Unheilige Allianzen. Black Metal zwischen Satanismus, Heidentum und Neonazismus. Münster: Unrast (3., korr. Aufl.).

Eck, Markus (2008/2009). "Streyf « [Rezension]. In: Pagan Fire 2 (The Legacy Chronicles), S. 67.

Edda (1983). Die Lieder des Codex Regius nebst verwandten Denkmälern. Teil 1: Text. Hg. v. Gustav Neckel. Heidelberg: Winter (5., verb. Aufl. von Hans Kuhn).

Edda Snorra Sturlusonar (1931). Hg. v. Finnur Jónsson. Kopenhagen: Gyldendal.

Grönbech, Wilhelm (1954). Kultur und Religion der Germanen. Bd. 1. Hg. v. Otto Höfler. Darmstadt: Wissenschaftliche Buchgesellschaft (5., unveränd. Aufl.).

Elflein, Dietmar (2010). Schwermetallanalysen. Die musikalische Sprache des Heavy Metal (= texte zur populären musik 6). Bielefeld: transcript.

Foster, Robert (2002): Das große Mittelerde-Lexikon. Ein alphabetischer Führer zur Fantasy-Welt von J.R.R. Tolkien. Bearb. u. ergänzt v. Helmut W. Pesch. Bergisch Gladbach: Bastei Lübbe.

Grimm, Jacob (2001/2003). Deutsche Mythologie. 2 Bde. Nach der Ausgabe von Elard Hugo Meyer neu hg. v. Helmut Birkhan (= Jacob Grimm und Wilhelm Grimm: Werke. Forschungsausgabe, Abteilung 1: Die Werke Jacob Grimms, Bd. 26/27). Hildesheim, Zürich, New York: Olms / Weidmann.

Hagen, Ross (2011). »Musical Style, Ideology, and Mythology in Norwegian Black Metal. "In: Metal Rules the Globe. Heavy Metal Music Around the World. Hg. v. Jeremy Wallach, Harris M. Berger und Paul D. Greene. Durham, NC, London: Duke University Press, S. 180-199.

Heesch, Florian (2009). »Black Metal-Sirenen. Mythenrezeption und Weiblichkeitsbilder bei Astarte. "In: History - Herstory. Alternative Musikgeschichten. Hg. v. Annette Kreutziger-Herr und Katrin Losleben (= Musik - Kultur - Gender 5). Köln: Böhlau, S. 389-404. 
Heesch, Florian (2011a). »Die Wilde Jagd als Identitätskonstruktion im Black Metal.«In: Eddische Götter und Helden. Milieus und Medien ihrer Rezeption. Hg. v. Katja Schulz (= Edda-Rezeption 2). Heidelberg: Winter, S. 335-365.

Heesch, Florian (2011b): »Thor im Metal und im Fantasy-Roman. Zur >Asgard Saga von Manowar und Wolfgang Hohlbein. "In: Heavy Metal Studies. Bd. 1: Lyrics und Intertextualität. Hg. v. Roman Bartosch. Oberhausen: Schmenk, S. 64-88.

Heesch, Florian (2012): »Metal for Nordic Men? Amon Amarth's Representations of Vikings «. In: Reflections in the Metal Void. Hg. v. Niall W.R. Scott. Oxford: Inter-Disciplinary Press, S. 167-182.

Helden, Imke von (2012). »Barbarians and Literature. Viking Metal and its Links to Old Norse Mythology. «In: Reflections in the Metal Void. Hg. v. Niall W. R. Scott. Oxford: Inter-Disciplinary Press, S. 155-166.

Herder, Johann Gottfried (1998). »Iduna, oder der Apfel der Verjüngung « [1796]. In: Ders., Werke in 10 Bänden. Bd. 8: Schriften zu Literatur und Philosophie 1792-1800. Hg. v. Hans Dietrich Irmscher. Frankfurt/M.: Deutscher KlassikerVerlag, S. 155-172.

Herold, Norbert (1976). »Kontinuität, historische. «In: Historisches Wörterbuch der Philosophie. Bd. 4. Hg. v. Joachim Ritter und Karlfried Gründer. Basel, Stuttgart: Schwabe, S. 1038-1042.

Hirschi, Caspar (2006). "Germanenmythos.«In: Enzyklopädie der Neuzeit. Bd. 4. Hg. v. Friedrich Jaeger. Stuttgart, Weimar: Metzler, S. 551-555.

Höpflinger, Anna-Katharina (2011). »Towards Babylon<. Mesopotamische Mythen im Metal.« In: Heavy Metal Studies. Bd. 1: Lyrics und Intertextualität. Hg. v. Roman Bartosch. Oberhausen: Schmenk, S. 45-63.

Jacobs, Rüdiger (2009). »Die Bedeutung nordischer Mythen in Richard Wagners Dramen-Konzept am Beispiel der >Ring<-Konzeption. «In: »Sang an Aegir«. Nordische Mythen um 1900. Hg. v. Katja Schulz und Florian Heesch (= Edda-Rezeption 1). Heidelberg: Winter, S. 179-211.

Johannesson, Ika / Klingberg, Jon Jefferson (2011). Blod Eld Död. En svensk metalhistoria. Stockholm: Alfabeta.

Kahn-Harris, Keith (2007). Extreme Metal. Music and Culture on the Edge. Oxford: Berg.

Macan, Edward (1997). Rocking the Classics. English Progressive Rock and the Counterculture. New York, Oxford: Oxford University Press.

Mørk, Gry B. (2011). "Why didn't the Churches Begin to Burn a Thousand Years Earlier?« In: Religion and Popular Music in Europe. New Expressions of Sacred and Secular Identity. Hg. v. Thomas Bossius, Andreas Hager und Keith KahnHarris. London, New York: Tauris, S. 124-144.

Moynihan, Michael / Søderlind, Didrik (2003). Lords of Chaos. The Bloody Rise of the Satanic Metal Underground. Port Townsend, WA: Feral House (2., überarb. und erw. Aufl.).

Mühlmann, Wolf-Rüdiger (2000). »Hamingja« [Rezension]. In: Rock Hard 18, H. 152, S. 98.

Mühlmann, Wolf-Rüdiger (2002). »Des Blutes Stimme« [Rezension]. In: Rock Hard 20, H. 187, S. 99.

Mühlmann, Wolf-Rüdiger (2003). »Riger. Provokation oder Missverständnis?« In: Rock Hard 21, H. 188, S. 62.

Schnurbein, Stefanie von (2007). »Neuheidentum und Fantasyroman.« In: Bilder vom Mittelalter. Eine Berliner Ringvorlesung. Hg. v. Volker Mertens und Carmen Stange (= Aventiuren. Sonderband). Göttingen: Vandenhoeck \& Ruprecht, S. 137-153. 
Schulz, Katja / Heesch, Florian (Hg.) (2009). "Sang an Aegir«. Nordische Mythen um 1900 (= Edda-Rezeption 1). Heidelberg: Winter.

Schulz, Katja (Hg.) (2011). Eddische Götter und Helden. Milieus und Medien ihrer Rezeption (= Edda-Rezeption 2). Heidelberg: Winter.

Stappert, Andreas (1998). »Der Wanderer« [Rezension]. In: Rock Hard 16, H. 135, S. 122.

Steuer, Heiko (2004). "Das >völkisch Germanische in der deutschen Ur- und Frühgeschichtsforschung. Zeitgeist und Kontinuitäten. «In: Reallexikon der Germanischen Altertumskunde. Ergänzungsbände, Bd. 34: Zur Geschichte der Gleichung "germanisch-deutsch«. Sprache und Namen, Geschichte und Institutionen. Hg. v. Heinrich Beck, Dieter Geuenich, Heiko Steuer und Dietrich Hakelberg. Berlin, New York: de Gruyter, S. 357-502.

Sweers, Britta (2002). »Die Fusion von traditioneller Musik, Folk und Rock - Berührungspunkte zwischen Popularmusikforschung und Ethnomusikologie. «In: Musikwissenschaft und populäre Musik. Versuch einer Bestandsaufnahme. Hg. v. Helmut Rösing, Albrecht Schneider und Martin Pfleiderer (= Hamburger Jahrbuch für Musikwissenschaft 19). Frankfurt/M.: Lang, S. 169-186.

Sweers, Britta (2005). Electric Folk. The Changing Face of English Traditional Music. Oxford, New York: Oxford University Press.

Trümpy, Hans (1973). »Einleitung. «In: Kontinuität, Diskontinuität in den Geisteswissenschaften. Hg. v. Hans Trümpy. Darmstadt: Wiss. Buchges., S. 1-10.

Walser, Robert (1993). Running with the Devil. Power, Gender, and Madness in Heavy Metal Music. Middletown, CT: Wesleyan University Press.

Wawn, Andrew (2005). "The Post-Medieval Reception of Old Norse and Old Icelandic Literature. "In: A Companion to Old Norse Icelandic Literature and Culture. Hg. v. Rory McTurk (= Blackwell Companions to Literature and Culture 31). Malden, MA: Blackwell, S. 320-337.

Widmaier, Tobias (2012). „Wir lieben die Stürme, die brausenden Wogen.“ In: Populäre und traditionelle Lieder. Historisch-kritisches Liederlexikon, http:// www.liederlexikon.de/lieder/wir_lieben_die_stuerme_die_brausenden_wogen (Zugriff am 28.3.2014).

Winter, Rainer (2001). Die Kunst des Eigensinns. Cultural Studies als Kritik der Macht. Weilerswist: Velbrück Wiss.

\section{Diskographie}

Adorned Brood (1996). Hiltia. Folter Records FR CD 005 (Reissue 2008: Black Bards Entertainment BBE $0015 \mathrm{AB}$ ).

Adorned Brood (2000). Asgard. Moonstorm Records RCO-018 (Reissue 2009: Black Bards Entertainment BBE $0025 \mathrm{AB}$ ).

Adorned Brood (2008). Noor. Black Bards Entertainment BBE 0018 AB.

Amon Amarth (2006). With Oden On Our Side. Metal Blade Records 3984-14584-2.

Bathory (1988). Blood Fire Death. Under One Flag FLAG 26 (Reissue 1993/2003: Black Mark BMCD 666-4).

Bathory (1990). Hammerheart. Noise International 4827-2-U (Reissue 1993: Black Mark BMCD 666-5).

Bathory (1991). Twilight Of The Gods. Black Mark BMCD 666-6.

Bathory (1996). Blood On Ice. Black Mark BMCD 666-12. 
Bjørnstad, Ketil (2001). Old. EmArcy 016 517-2.

Burzum (1997). Dauði Baldrs. Misanthropy Records AMAZON 013.

Grave Digger (2003). Rheingold. Nuclear Blast NB 1046-2.

Helheim (1995). Jormundgand. Karisma Records KAR017.

Helrunar (2005). Frostnacht. Lupus Lounge WOLF 014.

Helrunar (2007): Baldr ok Íss. Lupus Lounge WOLF 017.

Riger (1999). Hamingja. CCP Records 100200-2.

Sequentia (2002). The Rheingold Curse. A Germanic Saga of Greed and Revenge from the Medieval Icelandic Edda. Marc Aurel Edition MA 20016-

\title{
Filmographie
}

Walhalla (1986). Regie: Jeffrey James Varab und Peter Madsen. DVD. Starlight Film.

\begin{abstract}
German identity has often been constructed as Germanic/Teutonic. Norse mythology has played an important part in this construction as far as it has been constructed as "Germanic « mythology itself, or even as »German« since the early 19th century. Today, many heavy metal bands, even in Germany, make use of elements from Norse mythology. This article discusses relationships between such receptions of Nordic myths in heavy metal and constructions of Germanic identity. Besides song analysis the study investigates historical texts, draws on expert interviews and analyses of scene magazines. By referring to three exemplary German black and pagan metal bands (Helrunar, Adorned Brood, Riger), it can be shown that accesses to Norse mythology in metal vary from sophisticated adaptions of Eddic poems via cultural bricolage of diverse sources (mythology, fantasy, comic film etc.) to essentialist representations of Germanic-German identity.
\end{abstract}

